\title{
Oferta de frutas y verduras en instituciones educativas de un municipio del caribe colombiano
}

\author{
Offer of fruits and vegetables in educational institutions of a municipality of the Colombian Caribbean
}

\author{
Rusvelt Franklin Vargas-Moranth ${ }^{1 *}$ orcid.org/0000-0002-1014-0969 \\ Adalgisa Esther Alcocer-Olaciregui1 orcid.org/0000-0002-9509-8914 \\ Jorge Bilbao-Ramírez',2 orcid.org/0000-0001-7738-0740 \\ Jesús Ospino-Gutiérrez ${ }^{3}$ orcid.org/0000-0003-3334-8735
}

1 Universidad Metropolitana. Barranquilla, Colombia.

2 Universidad Libre. Barranquilla, Colombia.

3 Corporación Universitaria Reformada. Barranquilla, Colombia

\section{Resumen}

Introducción: Frente al creciente problema de obesidad infantil, en Colombia se han realizado avances en el marco legislativo, como la ley 1355 de 2009, la cual indica que las instituciones educativas deben ofertar frutas y verduras a los escolares. Objetivo: Describir la aplicación de la ley 1355 de 2009 frente a la oferta de frutas y verduras en instituciones educativas del municipio de Soledad-Atlántico. Materiales y métodos: Estudio descriptivo, transversal. Se encuestaron 18 directivos docentes: 12 coordinadores y 6 directores, mediante un formulario estructurado, y se hizo observación directa de la disponibilidad de frutas y verduras en las tiendas escolares, contrastando ambos aspectos. Resultados: 8/18 encuestados manifestó conocer la ley 1355; 11/18 indicaron que en su escuela existe un encargado de liderar programas relacionados con alimentación saludable; en $50 \%$ de las Instituciones se han adelantado programas de alimentación saludable; 15/18 (83,3\%) respondieron que sí hay disponibilidad de frutas y verduras en las tiendas escolares, pero esto se evidenció solamente en una de las 18 Instituciones (5,6\%). Conclusión: Se debe mejorar la disponibilidad de frutas y verduras en las tiendas escolares y lograr un empoderamiento de directivos, docentes, padres de familia y estudiantes, en aras de disminuir las cifras de obesidad.

Palabras clave: Frutas; verduras; escuelas; obesidad; alimentación saludable. (Fuente: DeCS, Bireme).

\begin{abstract}
Introduction: In response to the growing problem of childhood obesity, progress has been made in Colombia in the legislative framework; for instance, the law 1355 of 2009, which indicates that educational institutions should offer fruits and vegetables to school children. Objective: To describe the application of the law 1355 of 2009 facing the supply of fruits and vegetables in educational institutions in the municipality of Soledad, Atlántico. Materials and methods: A descriptive, transversal study was made. Twelve coordinators and six directors were surveyed through a structured form, and a direct observation was made of the availability of fruits and vegetables in the school stores, contrasting both aspects. Results: 8/18 respondents said they know law 1355; 11/18 indicated that there is a leader in their school to lead programs related to healthy eating, which have been advanced in $50 \%$ of the institutions; $15 / 18(83.3 \%)$ responded that there is availability of fruits and vegetables in school stores, but this was only evident in one of the 18 institutions (5.6\%). Conclusion: The availability of fruits and vegetables in school stores should be improved as well as the empowerment of managers, teachers, parents and students in order to reduce obesity numbers.
\end{abstract}


Key words: Fruits; vegetables; schools; obesity; healthy eating. (Source: DeCS, Bireme).

\section{Introducción}

La obesidad es considerada un problema de salud pública, presente tanto en países desarrollados como en los de menores ingresos(1). Colombia no es ajena a esta situación, la cual se vuelve más compleja cuando afecta a los niños y adolescentes(2), en ellos, los principales determinantes de la obesidad son la baja actividad física y la alimentación no saludable(3). A su vez, el consumo de alimentos hipercalóricos como pizzas, hamburguesas, perros calientes, fritos, alimentos de paquetes y bebidas azucaradas, entre otros $(4,5)$, suelen ser asequibles y baratos, rivalizando con una baja oferta de alimentos que son importantes para mantener el equilibrio energético, como son las frutas y las verduras(6).

Según la Organización Mundial de la Salud (OMS)(7), se calcula que la ingesta pobre de frutas y verduras es causal en todo el mundo de, aproximadamente $19 \%$ de los cánceres gastrointestinales, $31 \%$ de las cardiopatías isquémicas y $11 \%$ de los accidentes vasculares cerebrales, y alrededor de un $85 \%$ de la población mundial atribuye al escaso consumo de frutas $y$ verduras las enfermedades cardiovasculares, y un 15\% al cáncer.

La Organización de las Naciones Unidas para la Agricultura y la Alimentación $(\mathrm{FAO})^{(8)}$, recomienda un consumo mínimo de 400 gramos diarios de frutas y verduras, con el fin de prevenir enfermedades crónicas tales como las cardiopatías, el cáncer, la diabetes o la obesidad. Sin embargo, el consumo actual estimado de frutas y verduras es muy variable en todo el mundo, oscilando entre $100 \mathrm{~g} /$ día en los países menos desarrollados y aproximadamente 450 g/día en Europa Occidental ${ }^{(9)}$.

En el Departamento del Atlántico, el 80\% de las personas consume verduras, la mediana de consumo es baja: 56,5 gr/día; el 50\% de las verduras cultivadas corresponden a ahuyama, y las más consumidas son: cebolla de bulbo, tomate y zanahoria. De la misma forma, el 71,2\% consume frutas, con una mediana de consumo de $82,5 \mathrm{~g} /$ día; las principales frutas producidas en el departamento son: limón, mango y guayaba, y dentro de las más consumidas se destacan la naranja, el mango y el melón, con medianas de consumo mayores a $90 \mathrm{gr} /$ día(10). $^{(10}$

La OMS(11) estableció un plan de acción que proporciona una hoja de ruta para establecer, guiar $\mathrm{y}$ fortalecer iniciativas de vigilancia, prevención y tratamiento de las enfermedades no transmisibles como la obesidad, a partir de mejoras en la alimentación e incremento en los niveles de actividad física. En Colombia, en el año 2009 se promulgó la Ley 1355(12), la cual enfatiza la necesidad de promover estilos de vida saludable para contrarrestar la obesidad y sus consecuencias. Tomando en cuenta que en la mayoría de colegios privados y públicos, los estudiantes tienen acceso al almuerzo y alimentos que se venden en tiendas al interior de los centros educativos, esta Ley establece en el artículo $11^{\circ}$, que los colegios deben implementar estrategias tendientes a propiciar ambientes escolares que ofrezcan alimentación balanceada y saludable y fomentar la toma de decisiones adecuadas, que permitan a los estudiantes cualificar sus hábitos de vida, entre otros aspectos. El artículo 4 señala textualmente: "Los establecimientos educativos públicos y privados del país en donde se ofrezcan alimentos para el consumo de los estudiantes, deberán garantizar la disponibilidad de frutas y verduras".

El entorno infantil tiene una influencia determinante sobre el desarrollo de obesidad. Si en el colegio no se tiene claridad sobre los ambientes alimenticios sanos, eso sin duda puede afectar al menor, y por ello la ley 1355/2009 fijó las estrategias tendientes a promover una alimentación balanceada y saludable. Si la tienda escolar ofrece con la aquiescencia de las directivas de la Institución Educativa (IE), comidas no nutritivas y bebidas azucaradas, eso será lo que predomine en la dieta escolar del menor y fuera de la escuela buscará continuar con esta práctica alimenticia; incluso estos hábitos podrán trasladarse al 
entorno familiar. La publicidad desempeña un papel clave en este círculo que se puede romper si el menor está rodeado de ambientes nutricionales más sanos.

A través de un estudio previo realizado por el grupo investigador(13), se observó que en la mayoría de instituciones educativas del municipio de Soledad (Atlántico - Colombia), la oferta de frutas y verduras es pobre y no es claro el compromiso por parte de los directivos docentes, a pesar de la reglamentación existente en la Ley 1355 de 2009, por lo que se decidió llevar a cabo el presente estudio en el que se indaga por la aplicación de esta ley frente a la oferta de frutas y verduras en instituciones educativas del municipio de Soledad en el Departamento del Atlántico.

\section{Materiales y métodos}

Se llevó a cabo un estudio descriptivo, transversal, bajo el paradigma cuantitativo, en el que se aplicó una encuesta estructurada, cuya validez de contenido fue efectuada por consenso de un grupo de 10 expertos en metodología de la investigación, salud pública y nutrición, en el que $100 \%$ estuvo de acuerdo con la validez de cada pregunta, a directivos docentes de instituciones educativas públicas con ciclo de estudios secundarios $\left(6^{\circ}\right.$ a $\left.11^{\circ}\right)$ del municipio de Soledad, Atlántico, el cual según datos del Plan de Desarrollo Municipal(14) posee 30 instituciones con estas características, con 43 sedes distribuidas en 45 predios, $99 \%$ de ellas localizadas en el área urbana.

Se escogieron instituciones públicas, por ser estas las que principalmente deben implementar las normativas emanadas por el gobierno nacional, y por la accesibilidad otorgada por la Secretaría municipal de educación, a través de la cual se invitó a participar a los 30 colegios, pero solamente 18 directivos de estas Instituciones accedieron voluntariamente a participar y firmaron consentimiento informado, es decir, la proporción de escuelas participantes fue de $60 \%$.

Como criterio de inclusión, para la institución, se tuvo en cuenta aquellas de carácter público y que aceptaran participar, y como criterio de selección para el informante, se tuvo en cuenta solamente a directores y coordinadores, estos últimos, fueron encuestados si los primeros delegaban al proceso por no encontrarse, o estar ocupados en otras actividades.

La encuesta estuvo conformada por una sección concerniente a los datos de identificación de la escuela y del encuestado; en otra sección se indagó sobre la percepción del conocimiento de los lineamientos de la Ley 1355 de 2009, y aspectos relacionados con promoción de alimentación y estilos de vida saludable. Adicionalmente, para controlar posibles sesgos de información, los datos aportados por los encuestados fueron contrastados por observación directa, con una lista de chequeo, en las tiendas escolares. La información fue recolectada en los meses de febrero y marzo de 2016.

Los datos obtenidos fueron procesados mediante el programa informático SPSS Versión 22. La información es analizada mediante frecuencias relativas (porcentajes) y prueba de Fisher para determinar diferencias entre la proporción de coordinadores y directores.

\section{Consideraciones éticas}

La investigación fue aprobada por el Comité de ética de la Universidad Metropolitana de Barranquilla, y se desarrolló según la guía de Buenas prácticas clínicas (GCP), la Declaración de Helsinki y la Conferencia Internacional de Armonización (ICH); prevaleció el respeto a la dignidad y a la protección de los derechos y el bienestar de las personas. Según la Resolución 8430 de 1993, se consideró una investigación de "Riesgo inferior al mínimo". Los informantes participaron voluntariamente y diligenciaron consentimiento informado, previa aplicación de la encuesta.

\section{Resultados}

Participaron 12 coordinadores y 6 rectores. Al preguntarles si conocían los lineamientos de la Ley 1355 de 2009 con respecto a las IE, se encontró que $44,4 \%$ respondieron 
positivamente, y al desagregar esta respuesta según el cargo del encuestado, se observó que asintió una proporción mayor de coordinadores: 6/12 (50\%), frente a los directores: 2/6 (33\%), pero estas diferencias no fueron significativas (Fisher: 0,638) (Figura 1).

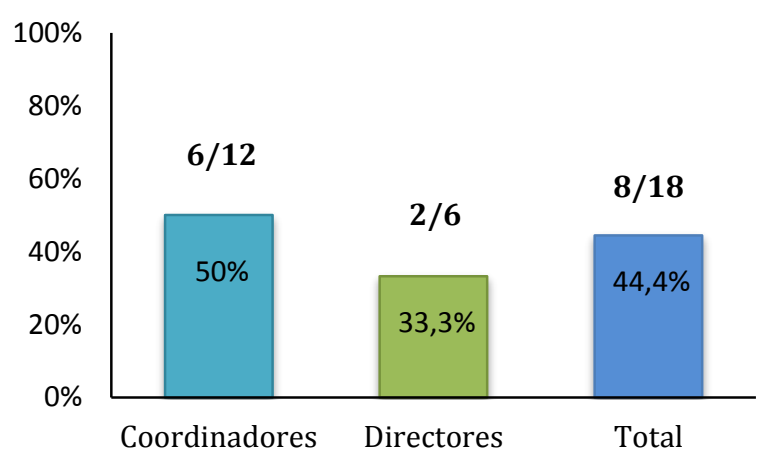

Figura 1. Conocimiento de los lineamientos de la Ley 1355 de 2009 en directivos docentes de las Instituciones Educativas del municipio de Soledad, Atlántico, 2016

Los encuestados manifestaron que en sus IE se ofrecen alimentos a los estudiantes a través de la modalidad de tienda o de comedor escolar, pero solamente una tercera parte indicó que se lleva a cabo un programa de educación alimentaria a nivel curricular. De la misma forma, la mitad de los encuestados indicó que en el último año se han llevado a cabo campañas para promover el consumo de frutas y verduras en su IE (Tabla 1).

Tabla 1. Aspectos relacionados con promoción de alimentación y estilos de vida saludable en las Instituciones Educativas participantes

\begin{tabular}{lc}
\hline En su Institución Educativa... & n=18 (\%) \\
\hline ¿Se ofrecen alimentos a los estudiantes? & $18 / 18(100 \%)$ \\
$\begin{array}{l}\text { ¿Se adelanta un programa de educación } \\
\text { alimentaria? }\end{array}$ & $6 / 18(33,3 \%)$ \\
$\begin{array}{l}\text { ¿Se han realizado campañas para } \\
\text { promover el consumo de frutas y } \\
\text { verduras? }\end{array}$ & $9 / 18(50 \%)$ \\
$\begin{array}{l}\text { ¿Existe una persona responsable de } \\
\text { promover estilos de vida saludable? }\end{array}$ & $11 / 18(61,1 \%)$ \\
\hline
\end{tabular}

Así mismo, 11/16 (61,1\%) de los encuestados indicó que en su IE hay una persona encargada de liderar la promoción de estilos de vida saludable (Tabla 1), que en la mayoría de los casos $7 / 11(63,6 \%)$ es el psicoorientador o "consejero escolar" cargo que suele ser desempeñado por un profesional en psicología o psicopedagogía (Figura 2).

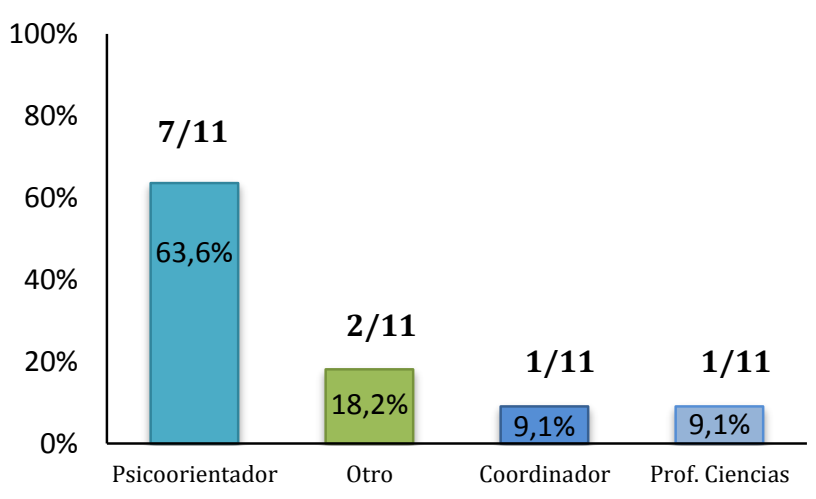

Figura 2. Cargo de la persona que lidera los programas de estilo de vida saludable en las Instituciones Educativas participantes

Finalmente, se preguntó a los directivos participantes si en su IE se ofrecen frutas y/o verduras en las tiendas escolares, y 15/18 $(83,3 \%)$ respondieron que sí. Sin embargo, al corroborar a través de una lista de chequeo mediante observación directa en el mismo día que se aplicó la encuesta, se encontró que solamente una IE ofrecía frutas para la venta en su tienda escolar, presentándose una enorme discrepancia entre la realidad y lo respondido por los participantes (Figura 3).

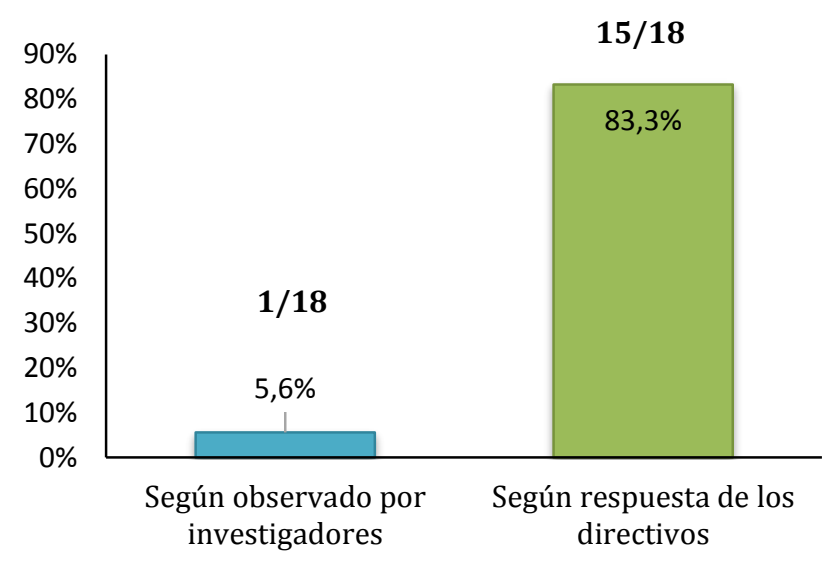

Figura 3. Instituciones Educativas que ofrecen frutas y/o verduras en las tiendas escolares, según lo 
observado por los investigadores Vs lo respondido por los directivos participantes

\section{Discusión}

No se tiene conocimiento que en el municipio de Soledad haya un programa liderado por las autoridades locales, para poder implementar los lineamientos de la ley 1355, a pesar que, según la un estudio liberado por la FAO en 2012, en la Región Atlántica colombiana, donde se ubica el municipio de Soledad, solamente $36 \%$ de las personas consumen las cantidades adecuadas de frutas y verduras, la mediana de consumo es de 82,5 gramos por día(15). Sin embargo, en el país se han dado experiencias exitosas, como la de ciudad de Cali(16), en la que la alcaldía municipal junto con el apoyo del sector privado, constituyeron las "tiendas escolares saludables", en donde se brinda una alimentación balanceada y saludable a los niños de la ciudad. De igual manera, en el departamento de Huila(17), la gobernación adoptó la política pública de seguridad alimentaria y nutricional, articulando dentro de sus componentes la "promoción de las tiendas saludables en donde se ofrezcan frutas y verduras" para establecer la estrategia "Escuela saludable".

En el presente estudio, el sesgo de información fue controlado mediante la contrastación entre lo expresado por el encuestado y lo observado por el encuestador. Se encontró que, menos de la mitad de los encuestados informaron que conocían los lineamientos de la Ley 1355 de 2009 con respecto a las IE; esto no quiere decir que el grado de conocimiento sea profundo o sea leve, ya que no se llevó a cabo una evaluación del conocimiento, sino que se asume que las respuestas provistas por los directivos docentes son verdaderas. En todo caso, llama la atención que una mayor proporción de Coordinadores hayan indicado que sí conocían la Ley, pues se esperaba que tanto ellos como los directores tuvieran conocimiento de esta, ya que ambos son responsables de implementar las acciones necesarias para el cumplimiento de la Ley en las instituciones educativas.

Sólo 33\% de los encuestados indicaron que en su IE se lleva a cabo un programa de alimentación escolar, con parámetros establecidos en el currículo, situación preocupante ya que todas las escuelas deberían incorporarlo a sus Proyectos Educativos (PEI), puesto que desde estos se genera un marco operativo estableciendo un norte y unas acciones específicas de manera sistemática y organizada para poder abordar efectivamente un programa de alimentación saludable(7). Desde el PEI se pueden establecer políticas para el desarrollo de campañas (como lo indicó el 50\% de los encuestados), en las que se intensifique la venta de frutas y se restrinja o prohíba el consumo de bebidas gaseosas, su reglamentación desde el PEI permitiría que las acciones no sean mediáticas y puedan generar un impacto a mediano y largo plazo.

Por otro lado, resulta impactante que, aunque la inmensa mayoría de encuestados indicaron que en su IE sí se ofrecen frutas y/o verduras en las tiendas escolares, la realidad fue diferente a esta respuesta, ya que solamente en una IE se pudo constatar que esta afirmación era verídica, es decir, la oferta de frutas y verduras resultó deficitaria, incluso inferior al $70 \%$ de escuelas que no disponen adecuadamente de oferta de frutas y verduras, encontrado por Castro et al.(18), pero superior al $0 \%$ encontrado por Velandia et al.(19), en Tunja. sin embargo, hay que tener en cuenta que, posiblemente el día de la visita no hubo oferta de frutas y verduras, ya que la observación hecha en las tiendas escolares por parte de los investigadores, fue de un solo día, y esto sugiere indagar, en una siguiente fase del estudio, por la cantidad, calidad y frecuencia, en la venta y oferta de frutas y verduras, según lo recomendado por Merlo et al.(20), quienes a través de un estudio longitudinal encontraron que casi $90 \%$ de las escuelas estadounidenses ofrecen frutas y verduras y casi el $80 \%$ dos o más tipos diferentes los cinco días de la semana escolar.

Es necesario que se incremente la oferta de frutas y verduras en las IE, puesto que se ha demostrado que trasciende los límites de la escuela y aporta una motivación especial a que los niños y adolescentes consuman esta clase de alimentos en sus casas y en otros ámbitos(21), y junto con estrategias encaminadas a mejorar los 
niveles de actividad física, se disminuya la prevalencia de obesidad.

\section{Conclusión}

Aunque la mayoría de directivos docentes manifiestan conocer los lineamientos de la Ley 1355, su implementación no es completa en las instituciones educativas que representan, por lo que se requieren llevar a cabo estrategias para su puesta en marcha.

\section{Conflicto de intereses}

Ninguno declarado por los autores.

\section{Referencias}

1. Malo M. Perspectives in the fight against obesity as a public health problem. Rev Peru Med Exp Salud Pública. 2017;34(1):5-6.

2. Han J, Lawlor D, Kimm S. Childhood obesity. Lancet. 2010;375(9727):1737-1748.

3. Liao Y, Siegel PZ, Garraza LG, Xu Y, Yin S, Scardaville M, et al. Reduced Prevalence of Obesity in 14 Disadvantaged Black Communities in the United States: A Successful 4-Year Place-Based Participatory Intervention. Am J Public Health. 2016;106(8):14421448.

4. Tanaka C, Reilly JJ, Huang WY. Longitudinal changes in objectively measured sedentary behaviour and their relationship with adiposity in children and adolescents: Systematic review and evidence appraisal. Obes Rev. 2014;15(10):791-803.

5. Colagiuri S. The obesity epidemic and sugar-sweetened beverages: a taxing time. Med J Aust. 2017;206(3):109110.

6. Chiasson MA, Scheinmann R, Hartel D, McLeod N, Sekhobo J, Edmunds LS, et al. Predictors of Obesity in a Cohort of Children Enrolled in WIC as Infants and Retained to 3 Years of Age. J Community Health. 2016;41(1):127-133.

7. Organización Mundial de la Salud. Estrategia Mundial Sobre Régimen Alimentario, Actividad Física y Salud. Resolución 57.17. Geneva: OMS; 2004.

8. Organización de las Naciones Unidas para la Alimentación y la Agricultura. 32a Conferencia Regional de La FAO Para América Latina y El Caribe. Buenos Aires: FAO; 2012.

9. Di Noia J, Schinke S, Prochaska J, Contento I. Application of the transtheoretical model to fruit and vegetable consumption among economically disadvantaged African-American adolescents: preliminary findings. Am J Heal Promot. 2006;20(5):342-348.

10. Olavarría S, Zacarías I. Obstaculizadores y facilitadores para aumentar el consumo de frutas y verduras en seis países de Latinoamérica. Arch Latinoam Nutr. 2011;61(2):154-162.
11. Organización Mundial de la Salud. Obesidad y Sobrepeso Nota Descriptiva $N^{\circ} 311$. Geneva: OMS; 2014.

12. República de Colombia. Ley 1355, de Estilos de Vida Saludables. Bogotá: Congreso de la Republica; 2009.

13. Vargas A, Alcocer O, García K, Villarreal, H. Consumo de frutas y verduras en adolescentes escolarizados del municipio-de Soledad, Atlántico. Abril-mayo de 2014. Rev Colomb Nutr y Metab. 2017;7(1):179.

14. Alcaldía Municipal de Soledad. Plan de Desarrollo Económico, Social y Urbano. 2008-2011. Soledad, Atlántico: Alcaldía Municipal; 2008.

15. Combariza J. Perfil Nacional de Consumo de Frutas y Verduras. Bogotá DC: Ministerio de Salud y Protección Social, FAO; 2013.

16. Ramírez M. Experiencia exitosa de Seguridad Escolar en Cali Colombia. En: USAID. Educación y Gestión Del Riesgo: Una Experiencia para Compartir. San José: USAID/OFDA; 2011.

17. Asamblea Departamental del Huila. Ordenanza 035 de 2010. Neiva, Huila: Asamblea Departamental; 2010.

18. Castro M, Ríos R, Úbeda C, Callejón R. Evaluación de menús ofertados en comedores escolares: comparación entre colegios públicos, privados y concertados. Rev Nutr. 2016;29(1):97-108.

19. Velandia N, Cárdenas L, Agudelo N. ¿Qué ofrecen las tiendas escolares y qué consumen los estudiantes durante el descanso? estudio en la ciudad de Tunja, 2009. Hacia la Promoción la Salud. 2011;16(1):99-109.

20. Merlo C, Brener N, Kann L, McManus T, Harris D, Mugavero K. School-level practices to increase availability of fruits, vegetables, and whole grains, and reduce sodium in school meals - United States, 2000, 2006, and 2014. MMWR Morb Mortal Wkly Rep. 2015;64(33):905-908.

21. Farris AR, Misyak S, Duffey KJ, Mann GR, Davis G, Hosig $\mathrm{K}$, et al. A comparison of fruits, vegetables, sugarsweetened beverages, and desserts in the packed lunches of elementary school children. Child Obes. 2015;11(3):275-280. 